\title{
Hasty Fault Diagnosis of a Rotating Machinery hinge on Stalwart Trippy Classifier with Robust Harmonized Swan Machine
}

\author{
Sumit Kumar Sar, Ramesh Kumar \\ Bhilai Institute of Technology Bhilai House, Durg, \\ Chhattisgarh 491001, India
}

\begin{abstract}
Monitoring with fault diagnosis of machineries are critically important for production efficiency and plant safety in modern enterprises. Along the process of fault diagnosis due to the addition of faulty signals, it is not an easy task to extract the exact representative features from the original signal. Accordingly, for making the vibration signal analysis more effective, there is a need to have the proper faulty feature extraction and moreover to have the proper estimation of spectral density for eminently producing stable decomposition results even if the signal contains missing values. Moreover, there is a difficulty to measure the correlation between the features with the existing fault diagnosis researches and also it considers more learning time as well as memory constraints which makes the learned concept difficult to understand for classifying the faulty features prominently. Thus to commensurate a perfect diagnosis, in this research a "Robust Harmonised Swan Machine (RHSM) with Stalwart Trippy classifier" is formulated in which the iterative estimation of each mode satisfying a self-consistency nature in decomposition method of RHSM which in turn resolves the missing sample problem eminently and aids reinforcement learning precisely which measures the correlation between the features to classify the faulty features extremely thereby it takes only less memory constraint with less learning time.
\end{abstract}

Keywords- Robust Harmonized Swan Machine, Stalwart Trippy Classifier, RMS, Crest factor, Kurtosis, feature Extraction

\section{INTRODUCTION}

\section{$\mathrm{M}$} achinery rotating mass often with very demanding performance standards, some of which are complex used in the industry today [1]. In that, Machine failure is the major challenge because a failure of a reliable lead-time is not able to predict, without effective evaluation consequently resulting in costly downtime can be devastating. Therefore, effective and efficient condition monitoring and fault diagnosis is essential for the industry $[2,3]$. However, the diagnosis of faults in rotating machinery is often a labour-intensive and timeconsuming. Effective and efficient fault diagnosis is always a challenging task for the technicians and plant diagnostics [4]. Faults in rotating machinery mainly include bearing defects, stator faults, rotor faults or eccentricity. According to statistics, nearly $50 \%$ of the faults of rotating machinery are related to bearings [5].

In order to ensure the high reliability of bearings and reduce the downtime of rotating machinery, it is extremely important to detect and identify bearing faults quickly and accurately [6]. The rolling bearing fault diagnosis method has always been a research hotspot. The vibration signals of rolling bearings often contain important information about the running state.
When the bearing fails, the impact caused by the fault will occur in the vibration signal [7, 8]. Therefore, the most common application of the bearing fault diagnosis method is to use the pattern recognition method to identify the fault by extracting the fault features of the bearing vibration signal [9].

However, the rolling bearing vibration signal is nonlinear and non-stationary. It is easily affected by the background noise and other moving parts during the transmission process, which makes it difficult to extract the fault features from the original vibration signal, and the accuracy of the fault diagnosis is seriously affected. Traditional time-frequency analysis methods have been used in bearing fault diagnosis and have achieved corresponding results, such as short-time Fourier transform and wavelet transform. However, all these methods have defects in the lack of adaptive ability for bearing vibration signal decomposition $[10,11]$. For complex fault vibration signals, relying only on subjectively setting parameters to decompose signals may cause the omission of fault feature information and seriously affect the performance of fault diagnosis.

After the feature extraction, the classifier should be utilized to realize automatic fault diagnosis in accordance with the machine learning algorithms, including pattern recognition and neural networks, require a large number of high-quality sample data [12]. In fact, bearing fault identification is controlled by the application environment. In reality, a large number of fault samples cannot be obtained. Therefore, it is crucial that the classifier can handle small samples and have good generalization ability. A support vector machine (SVM), proposed by Vapnik [13], is a machine learning method based on statistical learning theory and the structural risk minimization principle. Since the 1990s, it has been successfully applied to automatic machine fault diagnosis, significantly improving the accuracy of fault detection and recognition. Compared with artificial neural networks, SVM is very suitable for dealing with small sample problems, and has a good generalization ability. SVM provides a feasible tool to deal with nonlinear problems that is very flexible and practical for complex nonlinear dynamic systems. Besides, the combination of fuzzy control and a met heuristic algorithm is widely used in the control of nonlinear dynamic systems.

Bououden et al. proposed a method for designing an adaptive fuzzy model predictive control (AFMPC) based on the ant colony optimization (ACO) [14] and particle swarm optimization (PSO) algorithm [15], and verified the effectiveness in the nonlinear process. The Takagi-Sugeno ( $\mathrm{T}-$ 
S) fuzzy dynamic model has been recognized as a powerful tool to describe the global behaviour of nonlinear systems. $\mathrm{Li}$ et al. [16] deals with a real-time-weighted, observer-based, fault-detection (FD) scheme for T-S fuzzy systems. Based on the unknown inputs proportional-integral observer for $\mathrm{T}-\mathrm{S}$ fuzzy models, Youssef et al. [17] proposed a time-varying actuator and sensor fault estimation. Model-based fault diagnosis methods can obtain high accuracy, but the establishment of a complex and effective system model is the first prerequisite.

Yuan Xie and Tao Zhang [18] proposed the rotating machinery fault diagnosis with feature extraction algorithm based on empirical mode decomposition (EMD) and convolution neural network (CNN) techniques. The fundamental purpose of our newly proposed approach is to extract distinguishing features. Frequency spectrum of the signal obtained through fast Fourier transform process is trained in a designed CNN structure to extract compressed features with spatial information. To solve the non-stationary characteristic, we also apply EMD technique to the original vibration signals. EMD energy entropy is calculated using the first few intrinsic mode functions (IMFs) which contain more energy. With features extracted from both methods combined, classification models are trained for diagnosis.

Yong Lvet al. [19] proposed an approach to health degradation monitoring and early fault diagnosis of rolling bearings based on a complete ensemble empirical mode decomposition with adaptive noise (CEEMDAN)and improved multivariate multi-scale sample entropy (MMSE). The smoothed coarse graining process was proposed to improve the conventional MMSE. Numerical simulation results indicate that CEEMDAN can alleviate the mode mixing problem and enable accurate intrinsic mode functions (IMFs), and improved MMSE can reflect intrinsic dynamic characteristics of the rolling bearing more accurately. During application studies, rolling bearing signals are decomposed by CEEMDAN to obtain IMFs. Then improved MMSE values of effective IMFs are computed to accomplish health degradation monitoring of rolling bearings, aiming at identifying the early weak fault phase. Afterwards, CEEMDAN is performed to extract the fault characteristic frequency during the early weak fault phase.

Liang et al., [20] presented a rolling bearing fault diagnosis method based on ensemble local characteristic-scale decomposition (ELCD) and extreme learning machine (ELM)is proposed. Vibration signals were decomposed using ELCD, and numerous intrinsic scale components (ISCs) were obtained. Next, time-domain index, energy, and relative entropy of intrinsic scale components were calculated. According to the distance-based evaluation approach, sensitivity features can be extracted. Finally, sensitivity features were input to extreme learning machine to identify rolling bearing fault types.

Panget.al., [21] presented a bearing fault diagnosis method, namely an improved Hilbert time-time (IHTT) transform, by combining a Hilbert time-time (HTT) transform with principal component analysis (PCA). Firstly, the HTT transform was performed on vibration signals to derive a HTT transform matrix. Then, PCA was employed to de-noise the HTT transform matrix in order to improve the robustness of the HTT transform. Finally, the diagonal time series of the denoisedHTT transform matrix was extracted as the enhanced impulsive fault feature signal and the contained fault characteristic information was identified through further analyses of amplitude and envelope spectrums.

Li et.al. [22] Presents a bearing fault diagnosis method based on fully-connected winner-take-all auto encoder. The model explicitly imposes lifetime Sparsity on the encoded features by keeping only $\mathrm{k} \%$ largest activations of each neuron across all samples in a mini-batch. A soft voting method is implemented to aggregate prediction results of signal segments sliced by a sliding window to increase accuracy and stability.

\section{MOTIVATION AND CONTRIBUTION}

Motivated by the above discussion, the Condition observing and fault determination of Machinery are basically essential for creation productivity and plant security in current undertakings. At the point when a moving bearing shortcoming is feeble at a beginning time or at a low shaft speed, frail blame highlights are frequently implanted in foundation commotion. So it's anything but a simple undertaking to remove the agent features from the original signal. Numerous researchers have evaluated the issue of blame analysis taken from frail flags and have gained ground at the same time, the extraction of complex vibration signals is extremely troublesome. Many ways to deal with enhanced extraction have been proposed, by blend with the analytic devices: impartial systems, concealed Markov models, or solitary esteem disintegration. At times, great outcomes were acquired, yet these methodologies require the setting of a large number of parameters, but when the flag is very feeble or the commotion solid, these methodologies give poor outcomes. Henceforth we have to inspire the proposed system with appropriate component extraction technique with reasonable analytic instrument for making the eminent fault diagnosis as per the vibration signal analysis.

\section{ROBUST HARMONIZED SWAN MACHINE FOR FEATURE EXTRACTION AND STALWART TRIPPY ALGORITHM FOR FAULT DETECTION}

Condition monitoring and fault diagnosis of Machineries are critically important for production efficiency and plant safety in modern enterprises. When a rolling bearing fault is weak at an early stage or at a low shaft speed, weak fault features are often embedded in background noise. So it is not an easy task to extract the representative features from the original signal. Many scholars have reviewed the problem of fault diagnosis taken from weak signals and have made progress but, the extraction of complex vibration signals is very difficult Many approaches to improved extraction have been proposed, by combination with the diagnostic tools: neutral networks, hidden Markov models, or singular value decomposition. In some cases, good results were obtained, but these approaches 
require the setting of a great many parameters, and yet when the signal is quite weak or the noise strong, these approaches give poor results.

Hence in this research, we are proposing "Hasty Fault Diagnosis of a Rotating Machinery based on Stalwart Trippy Classifier with Robust Harmonised Swan Machine (RHSM)" for making the perfect fault diagnosis in vibration signal. The analysis of vibration signal is a preferred idea for fault diagnosis that is actually a classification task. For making that pattern recognition effectively, there is a need to have the proper faulty feature extraction. According to existing researches, the Harmonised Swan Machine (HSM) is the outstanding technique among Signal processing techniques. However, the main weakness of this machine is its high sensitivity to noise, and it also runs into the problem of mixing modes in which mode mixing causes the mixing of higher order components with the lower order components due to the Empirical Mode Decomposition(EMD) of this machine. Because of this mode mixing problem some wanted data will also be loosed. So removing some of the IMFs (Intrinsic Mode Function) can corrupt the data. To overcome this missing sample problem, we propose a Robust Harmonised Swan Machine(RHSM) with new decomposition method, termed Iterative Threshold VMD (Variation mode decomposition). Moreover, there is a difficulty to measure the correlation between the features with the existing fault diagnosis tool named "Trippy classifier "and also it considers more learning time and more memory constraints which makes the learned concept difficult to understand for classifying the faulty features prominently. In order to attain the perfect diagnosis, the eminent Neuro-Hybrid system is to be adapted in "Trippy classifier". For that we propose the Stalwart Trippy Classifier with the Dynamic Evolving Neuro-Fuzzy Inference System (DENFIS). By this reinforcement learning it exactly measures the correlation between the features to classify the faulty features eminently thereby it takes only less memory constraint with less learning time.

Fault detection is a fundamental procedure in scrutiny of vibration signal yielded from turning hardware. Vibration signals must be investigated top to bottom for that there is an expanded need to separate the features, for example, RMS, Kurtosis and Crest factor from each signal unit though acquiring features from every individual unit is gloomier. To examine every niche and corner, here we mounded a Robust Harmonized Swan Machine (RHSM), a direct nature roused system dependent on the character of Swan bird which has the stochastic nature to precisely extricate the destitute things dependent on the characteristic for feature extraction which thinks about each signal unit.

Formally, the useful features are precisely gotten by the guide of Hilbert Huang Transform which uses Iterative Threshold VMD (Variation mode decomposition) for successful IMF (Intrinsic Mode Function) extraction. The features are found out exclusively dependent on the faulty features utilizing DBN which at end yields the learned faulty features. For an effective fault diagnosis process there is an expanded need to precisely characterize faulty signals in lessened time which is finished with Stalwart Trippy classifier motivated from the prey getting conduct of Trippy fish. Stalwart Trippy fish evaluates all plausibility of prey habitation by its learning based seeking conduct. Essentially this classifier uses the random forest algorithm which makes a rundown of accessible yields yet it experiences precise prediction. With the end goal to handle the exact expectation is offered by the DENFIS which uses the knowledge based rule for prediction. In this way the precise order of faulty occurrence is finished utilizing Stalwart trippy classifier. The procedures utilized for every one of these steps is clarified and the general proposed architecture portrayed beneath. The block diagram is depicted below in figure 1

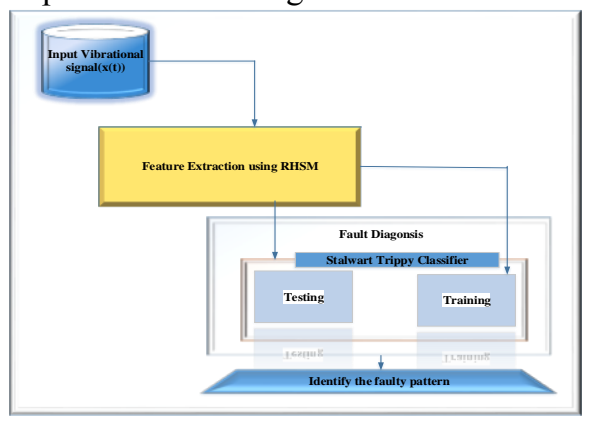

Figure 1: Basic Block Diagram of Proposed Methodology

This proposed architecture shows Robust Harmonized Swan Machine withStalwart TRIPPY Algorithmfor feature extraction and Fault Diagnosis which aims at improving automatic identification of faults accurately. At first the framework starts by gathering machine created crude data from proper areas by the data acquisition technique. The gathered crude vibrated data can be extremely loud, and furthermore be exposed to different natural contaminants. In order to detect features effectively notifying and eradicating noise, Robust Harmonized Swan Machine was developed. This feature extraction method consequently pre-prepared the crude data with the assistance of rapid preparing and uses the benefit of DBN accordingly focusing on the extraction of just required RKC features. RHSM decides the features of the original signal for each instantaneous frequency independent of signal type by the greedy layer wise learning empowering quick and dynamic sorts without repetition and clamour. Each signal's RKC features are resolved and learnt which holds the faulty signal information. After this procedure with the end goal to recognize the fault diagnosis naturally, a scholarly example arrangement technique named Stalwart trippy classifier is presented. At long last fault and normal feature are classified which depends on the pattern matching idea and the predicted output is at long last recovered. The process is as follows,

\section{A. Data Acquisition}

Data acquisition is the underlying advance of fault diagnosis, where machine singularities are estimated for further investigations and this paper is centered on vibrated signal-based fault diagnosis as described in equation (1).

$$
B_{i}(t)=\left\{B_{1}(t), B_{2}(t) \ldots \ldots . . B_{m}(t)\right\}
$$


Let $\mathrm{B}_{i}(t)$ is the arrangement of data of vibrated unique signal from other mechanical segments of the machine. In turning apparatus, any element can be ascertained on the crude vibration data and the data must be pre-processed early, however in this work the crude data is consequently preprocessed with the assistance of our proposed feature extraction procedure. After data acquisition the vibrated signals are the passed to the feature selection process.

\section{B. Robust Harmonized Swan Machine (RBSH) for Feature Selection}

At first vibrated data are sending to the feature extraction stage for expulsion of vibrated commotion and exact extraction of proposed features. With the end goal to remove the precise features we have created most slam against strategy RHSM which is encircled out dependent on the motivation of the exact specific detachment of needy components by a swan behavior. Here the needy components to be the strong highlights RKC and the particular procedure is finished by using DBN thusly for exact choice HHT is used as like the stochastic social of swan flying creature. The RHSM prepreparing strategy treats each consecutive pair of layers in the learning procedure, whose joint likelihood is characterized as,

$$
P_{h, u}(h, v)=\frac{1}{j(\omega)_{h, v}} \cdot e^{\left(B_{i}(t) w h+v^{T} b+a^{T} h\right)}
$$

The above equation (2) portrays the RBM (Restricted Boltzmann Machine) that has deep learning nature which comprised in each layer $h$ and shape the underlying DBN structure, and after that the regressive adjusting is connected to consistent variable v. DBN utilize the marked information train the conditional probability which has indistinguishable frame from that in DBN layer, and the error spread start to finish to tweaking the system. Contrasting the yield estimation of the deep system yield unit with the real esteem, figure the error esteem caused by the weight $\mathrm{w}$. The error esteem is transmitted back to get the error caused by each layer, and afterward the effective weight estimation procedure to extricate the fault component is computed. Presently it is important to calculate weight with appropriate inclination $b$ to alleviate the preparation error which is given in

$$
P_{h, u}(h, v)=\frac{1}{j(\omega)_{h, u}} \cdot e^{\left(B_{i}(t) w h+(v-b)^{T}(v-b)+a^{T} h\right)}
$$

The RBM parameters can be efficiently trained in an unsupervised fashion by maximizing the likelihood of the joint probability in equation (2)

$$
K=\Pi_{t} \sum_{h} P_{h, u}(h, B(t))
$$

This $\mathrm{K}$ is over training samples of vibrated signal $B(t)$. With the end goal to separate the IMFs of a perplexing signal in feature extraction, the training phase of RHSM calculation receive the iterative threshold VMD technique to decide the mono part of the first vibrated crude signal. Regardless of whether a signal contains missing qualities, this iterative threshold VMD break down a flag into meaningful IMFs successfully. That means, by the coupling of there sholding of period gram and an iterative estimation of every mode fulfilling a self-consistency nature in this decomposition method has settled that missing example issue prominently. After iterative threshold VMD, a signal B (t) can be expressed as in equation 5 ,

$$
B(t)=\sum_{k=1}^{N} z_{k}(t)+y_{n}, k=1,2, \ldots \ldots . . N
$$

To precisely extricate the missing example in the flag, consider the ceasing rule is used for foundation of combination for some $\delta>0$ which is given beneath,

$$
\sum_{K} \frac{\left\|z_{k}^{(m+1)}-z_{k}^{m}\right\|_{2}^{2}}{\left\|z_{k}^{m}\right\|_{2}^{2}}<\delta
$$

Where is the its decomposed IMF of the signal, is the uproarious signal. Here the iterative threshold VMD decides the mono segment of the first signal independently however there is an all-inclusive need to analyze each mono component separately dependent on its instantaneous recurrence, so the viable features can be gotten from each individual of vibrated signal and likewise what's more this decomposition technique forms results with some constrained measure of clamor, which is additionally to be handled. Above expressed issues are handled by taking the Hilbert Haung transform which impacts a specific preprocessing of the useful signal features acquired. Accordingly, HHD is connected on the two sides of Eq. (5), the Hilbert Haung range of B (t), Q $(\omega, t)$, might be done by the accompanying condition (6):

$$
Q(\omega, t)=S \mathrm{e} \sum_{i=1}^{n} B_{i}(t) e^{l \int \omega_{i}(t) d t}
$$

Where Se is the operator of real part, $B_{i}(t)$ and $\omega_{i}(t)$ denote the functions of the amplitude and instantaneous frequency, respectively. Note that the residual term $y_{n}$ in Eq. (5), which involves next to no vitality of the signal, is overlooked that is the commotion is maintained a strategic distance from which is equal to preprocessing of signal by using the RHSM nature dependent on eqn (7), which thus diminishes the time factor for extraction of faulty features.

Accordingly, the marginal spectrum of Hilbert-Huang transform which extract RKC features can be defined by an integrated spectrum with respect to time as in equation (8)

$$
Q(\omega)=\int_{0}^{T} Q\left(\omega, t_{(\text {RMS }, \text { Crest Factor }, \text { Kurtosis })}\right) d t
$$

Where $T$ is no of the features of $B(t)$ and $Q(\omega, t)$ can precisely define the extraction of efficacious faulty feature such as RMS, Kurtosis, and Crest of each individual IMF mono component with characterized time interim. The benefit of our extraction strategy with the assistance of HHT is to acquire all component extracted within instantaneous frequency restrict without clamour presence.

To such an extent that ascertaining IMF and Residue utilizing RHSM, the machine commotions are destroyed and crude information are moulded naturally with the assistance of RHSM Training. In this way all in all element Extraction by Robust Harmonized Swan Machine (RHSM) is the procedure of definitely removing required features for the faulty flag 
from the crude vibrated flag. The general design of Robust Harmonized Swan Machine is depicted underneath fig 2.

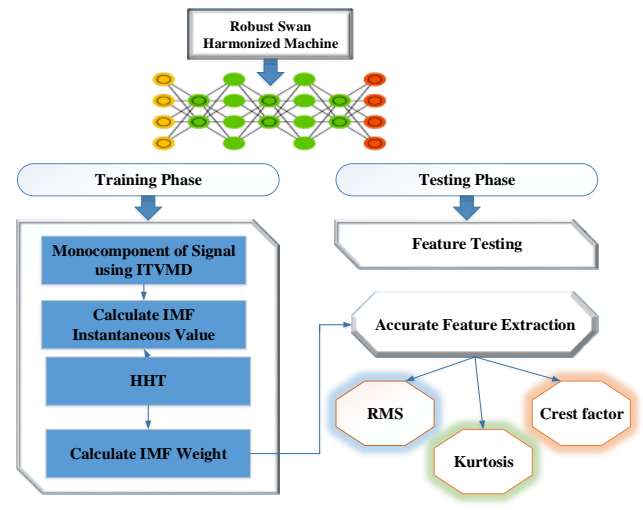

Figure 2: Robust Harmonized Swan Machine schematic architecture

Ordinarily, the factors, for example, RMS, Kurtosis, and Crest factor are believed to be noticeably anticipated criteria of a faulty signal when contrasted and typical vibrated signals. Along these lines by breaking down the RKC features it is less demanding for thumping out of the faulty signals without random looking which is the purpose behind deeply learning the three parameter RKC. This RKC translates as the way to pass judgment on the faulty signals happened. The depiction of RKC Features are given underneath

\section{a. $R M S$}

The root mean square (RMS) estimation of a vibration signal is a time analysis feature that is the proportion of the power content in the vibration signature. This feature regards track the general clamor level, yet it won't give any data on which segment is fizzling. It very well may be exceptionally compelling in identifying a noteworthy out-of-balance in pivoting frameworks. The following is the condition that is utilized to ascertain the root mean square estimation of a prepared information arrangement, $\mathrm{u}(\mathrm{t}) \mathrm{n}$ over length $\mathrm{N}$.

$$
R M S=\sqrt{\frac{1}{N}} \times \sum_{n=1}^{n} B(t)_{n}^{2}
$$

\section{b. Kurtosis}

Kurtosis is characterized as the conveyance and measures the relative peakedness or levelness of a dissemination when contrasted with a typical dispersion. Kurtosis gives a proportion of the measure of the tails of appropriation and is utilized as a marker of significant crests in an arrangement of information. As a rigging wears and breaks this feature should signal a blunder because of the expanded level of vibration. The condition for kurtosis is given by:

$$
\text { Kurtosis }=\frac{\sum_{n=1}^{N}[B(t)-\mu]^{4}}{N \times\left(\sigma^{2}\right)^{2}}
$$

where $v(t)$ is the raw time series at point $\mathrm{n}, \mu$ is the mean of the trained data, $\sigma^{2}$ is the variance of the data, and $\mathrm{N}$ is the total number of data points.

\section{c. Crest Factor}

A superior measure is to utilize "crest factor" which is characterized as the proportion of the pinnacle level of the info signal to the RMS level. In this manner, tops in the time arrangement signal will result in an expansion in the crest factor esteem. The following is the condition for the crest factor:

$$
\text { Crestfactor }=\frac{\text { Peak Level }}{R M S}
$$

At last features are extricated consummately without commotion and excess. The fundamental preferred standpoint of our proposed highlight extraction system is the flexibility of all signal kind because of the joint probability distribution parameter of RBM. Subsequent to getting features, an astute example order is expected to satisfy the defective conclusion naturally by the gathering of each one of those separated features. In this work Stalwart TRIPPY Algorithm proposed to satisfy the blame finding naturally by gathering every one of those removed monochrome features which is accomplished by the predictable ensemble nature of the classifier and is proposed underneath.

\section{Fault Diagnosis Using Stalwart Trippy Algorithm}

The Precise classification is accomplished in our work by using most spearheading nature motivated classifier absolved as stalwart trippy classifier dependent on the profound looking and exact prescient nature of trippy angle though this classifier offers a profound learning or seeking by methods for the randomized character of RF (Random forest) additionally prescient capacity by methods for DENFIS with information based earlier judging. In this manner once the scholarly flawed highlights are feeder to this classifier which investigation profoundly and offers precise determination which in turn isolates the characterized faulty signals.

Primarily the harvest outcomes $\mathrm{L}_{\mathrm{i}}$ (1) and $\mathrm{L}_{\mathrm{i}}$ (2) of the trained HSM should be fed into the trippy classifier which compromises deep learning by means of RF. The data fusion training of the faulty features is defined as

$$
\begin{aligned}
& {\left[\begin{array}{l}
i \quad{ }^{\circ(1)} \\
\operatorname{arq}_{i} \max K_{i}^{(1)}=\operatorname{arq}_{i} \max j\left(\omega_{i}\right) \\
i \quad \operatorname{arq}_{i} \max K_{i}^{(2)}=\operatorname{arq}_{i} \max j\left(\omega_{i}\right)
\end{array}\right]_{\text {Where } \mathrm{i}=1,2,3 \ldots \mathrm{m},}} \\
& \text { where }\left\lfloor j\left(\omega_{i}\right)=j\left(\omega_{1}\right)+j\left(\omega_{2}\right)+\ldots . .+j\left(\omega_{m}\right)\right\rfloor
\end{aligned}
$$

The Learning Features $\mathrm{K}_{\mathrm{i}}\left(j\left(\omega_{i}\right)\right)$ is imported from equation 7 that consist of RKC, which is utilized for diagnosing the fault.

$$
K_{i}=\left[K_{1}^{(1)}, K_{2}^{(2)}, \ldots, K_{m}^{k}\right]
$$

The above equation (13) is utilized to develop a tree with various bootstrap test from unique information utilizing a tree classification calculation. Where ' $\mathrm{m}$ ' is the quantity of highlights which are removed from RHSM. After the timberland is framed, protest that should be prepared is put down under every one of the tree in the forest for preparing. The preparation highlights are depicted in the equation (14)

$$
\omega_{i}=K_{i} \max j\left(\omega_{i}\right)
$$

Following, precise forecast procedure of the Stalwart trippy classifier after profound learning is finished by using the 
Neuro fuzzy interference system which uses information based example recognizable proof for the expectation of vibrated blame signal. Here we are using a DENFIS demonstrate which utilizes a dynamic Takagi-Sugeno fuzzy inference system. Notwithstanding powerfully making and refreshing fuzzy rules amid the learning procedure, the fluffy guidelines that take part in the derivation for each new info vector are progressively browsed the current fuzzy principle set contingent upon the situation of the current information vector in the input space. DENFIS is made out of $m$ fuzzy standards showed as pursues:

if $x 1$ is $R m 1$ and $x 2$ is $R m 2$ and ... and $x q$ is $R m q$, then $y$ is $f m(x 1, x 2, \ldots, x q)(15)$

where "xj is Rij", $i=1,2, \ldots m ; j=1,2, \ldots q$, the overall output will be

$$
y^{0}=\frac{\sum_{i=1}^{m} w_{i} f_{i}\left(x_{1}^{0}, x_{2}^{0}, \ldots . x_{q}^{0}\right)}{\sum_{i=1}^{m} w_{i}}
$$

Where, ${ }_{w_{i}}=\prod_{j=1}^{q} S_{i j}\left(x_{j}^{0}\right) ; i=1,2, \ldots \ldots . . m, j=1,2, \ldots \ldots q$

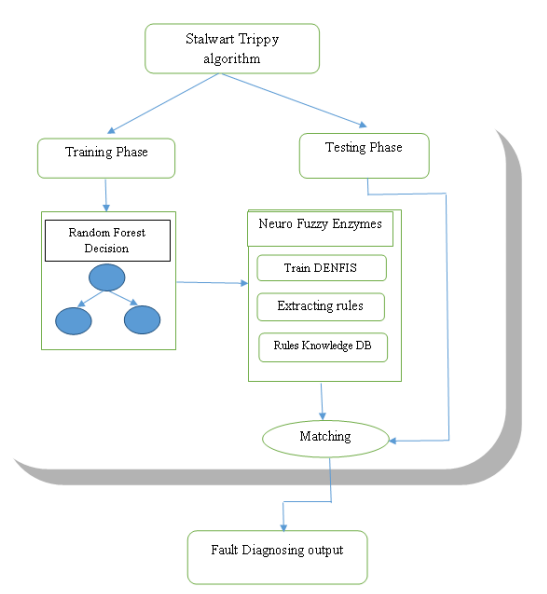

Figure 3: Stalwart Trippy Classifier for fault Diagnosing

In this manner by the use of proficient feature extraction by using RHSM and the keen pattern recognition by methods for Stalwart trippy classifier correctly characterizes blame with the help of learning based feature extraction. This is the sufficiently bright process because of the expellation of preprocessing and precise prediction process which wipes out the regarded use of time. The outcome approval in the underneath segment will be an additional verification for the productivity of the work. Thus efficient outputs in fault diagnosis of vibrating signals are done by utilizing this framework is discussed following in the result and discussion section stated below,

\section{RESULTS AND DISCUSSION}

The proposed technique is described in previous section 3 and in this section the detail explanation and its performance is analyzed. The proposed method is implemented in the working platform of MATLAB with the following system specification.

$\begin{array}{ll}\text { Platform } & \text { : MATLAB 2015a } \\ \text { OS } & \text { : Windows } 8 \\ \text { Processor } & \text { : Intel core i5 } \\ \text { RAM : 8 GB } & \text { RAM }\end{array}$

\section{A. Data collection}

Dataset was collected on a single stage reciprocating type air compressor placed at the Department of Electrical Engineering Workshop. Specifications of the air compressor are as follows:

- Air Pressure Range: 0-500 lb/m2, 0-35 Kg/cm2

- Induction Motor: $5 \mathrm{HP}, 415 \mathrm{~V}, 5 \mathrm{Am}, 50 \mathrm{~Hz}, 1440 \mathrm{rpm}$

- Pressure Switch: Type PR-15, Range 100-213 PSI

Dataset comprises of multiple states which includes healthy state and 7 faulty states namely Leakage Inlet Valve (LIV) fault, Leakage Outlet Valve (LOV) fault, Non-Return Valve (NRV) fault, Piston ring fault, Flywheel fault, Rider belt fault, and Bearing fault. To collect recordings from all these states, faults were seeded into the air compressor.

\section{B. Simulation Result}

The vibrated bearing fault raw dataset are plotted in the given below

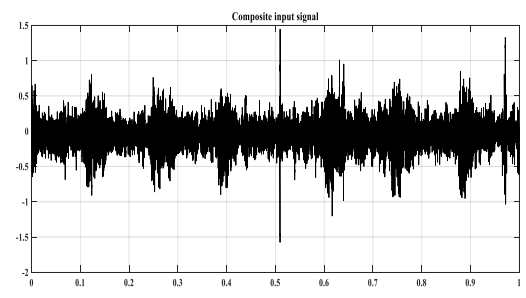

Figure 4: Original raw bearing vibrated signal of bearing

The vibrated signal is concentrating on the feature extraction like RMS, kurtosis, Crest factor. In this Paper Robust Harmonized Swan Machine is used for exact extraction of feature. ITVMD decides the mono part of unique signal. Our proposed natural mode capacity of the original signal is depicted in fig 5,6 and 7 . 


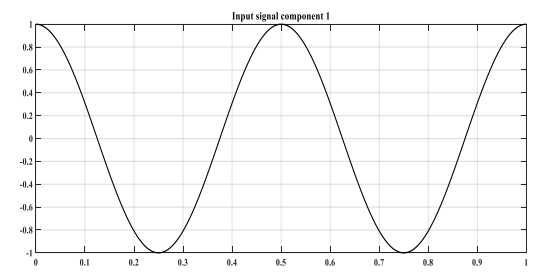

Figure 5: IMF 1 values of original signal

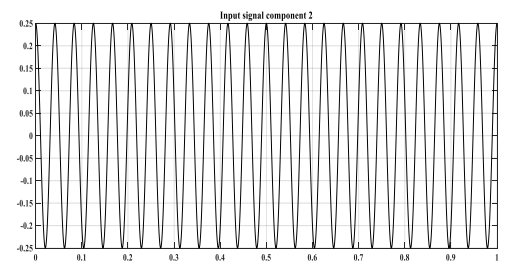

Figure 6: IMF 2 values of original signal

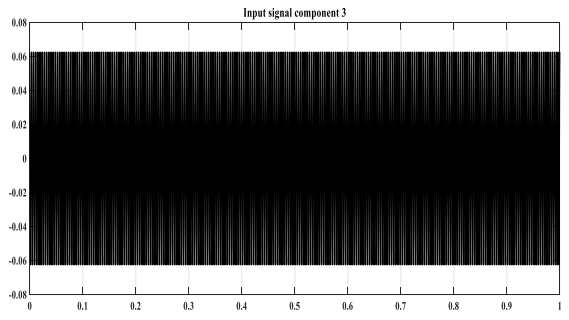

Figure 7: IMF 3 values of original signal

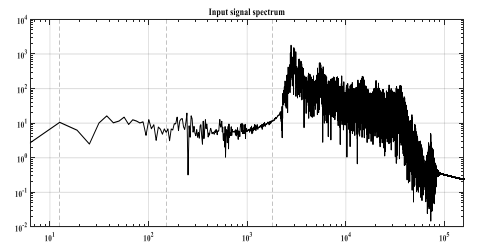

Figure 8: Spectrum of the input signal

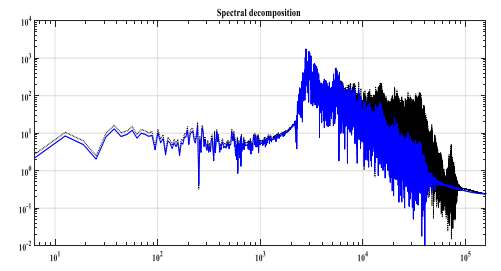

Figure 9: ITVMD spectral decomposition

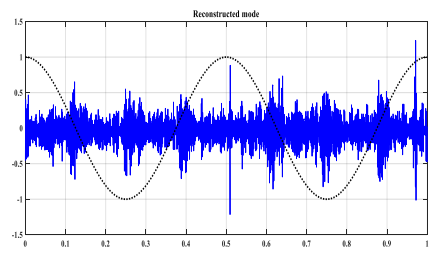

Figure 10: ITVMD Reconstructed modes

After ITVMD technique the instantaneous frequency is calculated using HilbertHaung Transform. In this below figure
10 shows that the spectrogram of HHT of instantaneous frequency signal.

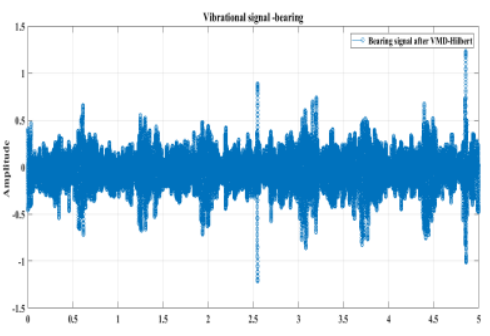

Figure 11: ITVMD based Hilbert-Huang transform

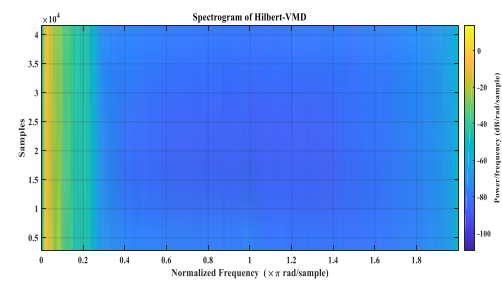

Figure 12: Spectrogram of ITVMD- Hilbert Haung Transform

After that procedure some flawed signal are accessible in the above data. With the end goal to evade this issue RHSM is used for profound learning. The learning conduct of RHSM is utilized to extricate the required feature for blame finding in exact path with the assistance of IMF computation dependent on the idea of RHSM which is broadly utilized monochrome of individual recurrence signal. At long last exact commotion features are disposed of and our required features, for example, RKS are precisely separated inside the less time. After this procedure our proposed feature extraction are portrayed in the underneath fig $13,14,15$.

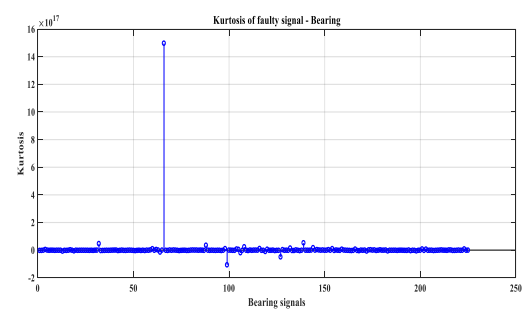

Figure 13: Kurtosis of bearing

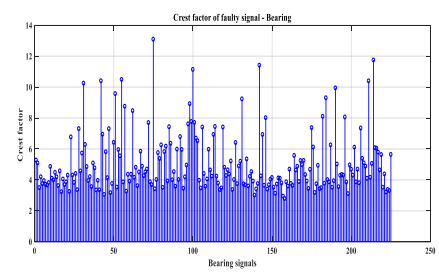

Figure 14: Crest factor of bearing 


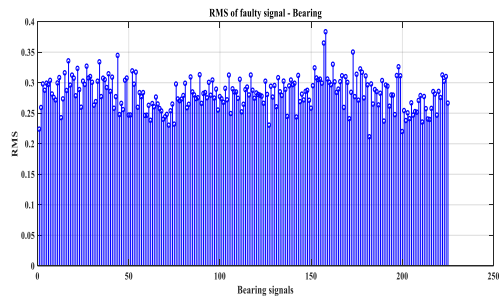

Figure 15: RMS of bearing

With the end goal to anticipate the precise vibrated signal, the insightful based example order is required, so in this examination paper center around Stalwart TRIPPY for predict the exact defective signal consequently. The random forest decision tree doesn't anticipate the exact signal since it has apriori learning less nature

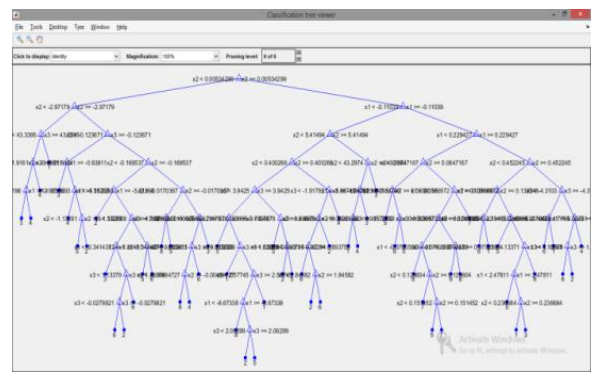

Figure 16: Stalwart Trippy algorithm decision Tree

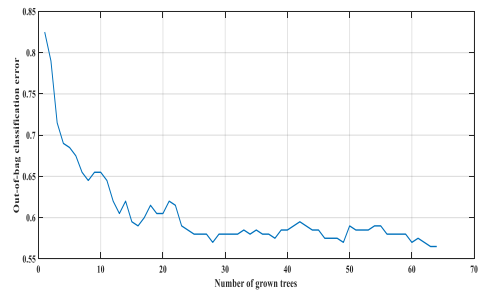

Figure 17:Proposed accurate faulty signal detection based on decision Tree

In the above fig 17 depicts the faulty signal detection in the revolution machine utilizing Stalwart TRIPPY Classifier.Finally the precise conduct of faulty state are distinguished in best way. Our proposed calculation has information based standard programmed detection is accustomed to diagnosing the faulty signal.

\section{Comparision Analysis}

Comparison was made by proper analysis of computation time, accuracy,Diagonosis Accuracy,Testing Prediction Time and Diagonosis Noise are described below section.

\section{a. Computation time}

Computation time amid feature extraction is characterized as the time required for separating the fundamental features from crude vibrated information. The calculation equations are depicted underneath.

\section{Computation Time $=$ Starting Time of featureextraction - Ending Time of featureextraction \\ TABLE 1 \\ COMPUTATION TIME OF PROPOSED TRANSFORM AND EXISTING TRANSFORM}

\begin{tabular}{|c|c|c|}
\hline Sl.no & Algorithm & $\begin{array}{c}\text { Run } \\
\text { Time }\end{array}$ \\
\hline 1 & TDSP & 0.034 \\
\hline 2 & Fast flourier Transform & 0.013 \\
\hline 3 & Morlet wave let Transform & 0.072 \\
\hline 4 & Discrete wave let transform & 0.092 \\
\hline 5 & Short time Fourier transform & 0.648 \\
\hline 6 & Wigner wile Distribution & 0.008 \\
\hline 7 & Pseudo- Wigner wille Distribution & 2.120 \\
\hline 8 & Auto correlation & 0.028 \\
\hline 9 & Updated more let Transform & 0.029 \\
\hline 10 & Convolution with sine & 0.012 \\
\hline 11 & S-transform & 5.328 \\
\hline 12 & Proposed & 0.019 \\
\hline
\end{tabular}

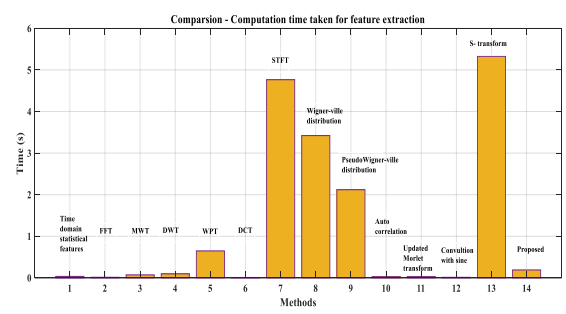

Figure 18: comparison of proposed and existing transform for feature extraction

In the above fig 18 exhibits the normal calculation time per recording for processing each change and its comparing highlights. These qualities were found by averaging the calculation time more than 1000 chronicles, which depicts the computation time for existing change for dissecting the chosen includes, those utilizing FFT, WPT, DCT, WVD, STFT the vales are portrayed in table 1 and our proposed highlight extraction which use preparing with ITVMD based HHT achieve the lower computation time when contrasted with existing works.

\section{b. Accuracy}

The accuracy of a test is its capacity to separate the faulty and healthy condition effectively. To gauge the accuracy of a test, we ought to figure the extent of genuine positive and genuine negative in all assessed cases. Numerically, this can be expressed as:

$$
\text { Accuracy }=\frac{a+d}{a+d+c+b}
$$

Where, True positive (a) = the number of features correctly identified as faults,

False positive $(\mathrm{c})=$ the number of features incorrectly identified as faults,

True negative $(\mathrm{d})=$ the number of features correctly identified as Normal,

False negative $(b)=$ the number of features incorrectly identified as Normal. 
TABLE 2

ACCURACY COMPARISON OF VARIOUS BEARING AND PROPOSED BEARING

\begin{tabular}{|l|l|l|}
\hline Sl.no & Algorithm & Total $(\mathbf{s e c})$ \\
\hline 1 & KNN & 86.67 \\
\hline 2 & PNN & 90 \\
\hline 3 & RBN & 96.67 \\
\hline 4 & PSO-SVM & 96.67 \\
\hline 5 & PROPOSED & $\mathbf{9 8 . 6 7 5}$ \\
\hline
\end{tabular}

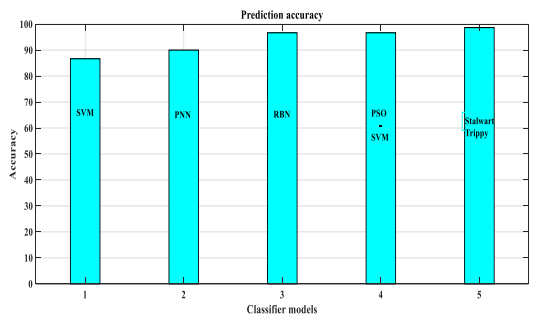

Figure 19: Accuracy comparison for various bearing method of existing and proposed

In the above fig 19 contributions the Accuracy prediction time per recording for processing each change and its relating fault diagnosis, which depicts the accuracy for existing and breaking down the diagnosing the fault, those utilizing KNN, PNN, RBN, PSO-SVM, and proposed the qualities are portrayed in table 2 and our proposed stalwart Trippy algorithm, which achieve the higher accuracy when contrasted with existing algorithm.

\subsubsection{Testing prediction Time}

The testing prediction time is defined as the time taken to predict the precise fault.

TABLE 3

OVERALL PREDICTION USING EXISTING AND PROPOSED

\begin{tabular}{|l|l|l|}
\hline Sl.no & Algorithm & Total (sec) \\
\hline 1 & KNN & 0.51 \\
\hline 2 & PNN & 0.064 \\
\hline 3 & RBN & 20.6307 \\
\hline 4 & PSO-SVM & 0.05033 \\
\hline 5 & PROPOSED & 0.061 \\
\hline
\end{tabular}

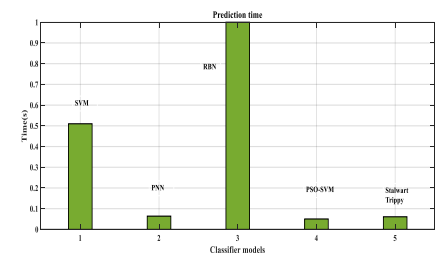

Figure 20: overall prediction time using existing and proposed

In the above fig: 20 that portray the general prediction examination of our proposed algorithm and existing algorithm, for example, KNN, PNN, RBN, PSO-SVM and proposed esteems are plotted in the Table:3. At long last our proposed Stalwart Trippy classifier accomplish less prediction time when contrasted with all other existing algorithm and furthermore accurately determine the fault to have 0.061 secs which is very higher. This time is worthy since we do preparing twice and we are accepting this less time.

\subsubsection{Comparison of Diagnosis Accuracy}

The Diagnosis Accuracy is characterized as the general likelihood that a fault will be effectively arranged dependent on the learning test informational collection. The Diagnosis Accuracy is portrayed underneath

$$
\text { Diagnosis Accuracy }=\frac{a+d}{a+b+d+c}
$$

TABLE 4

COMPARISON OF DIAGNOSIS ACCURACY PROPOSED AND EXITING METHOD

\begin{tabular}{|c|c|c|c|c|c|c|}
\hline SI.No & $\begin{array}{c}\text { Features } \\
\text { Algorithm }\end{array}$ & $\begin{array}{c}\text { Classifier } \\
\text { Algorithm }\end{array}$ & $\begin{array}{c}\text { No.of } \\
\text { training } \\
\text { sampes }\end{array}$ & $\begin{array}{c}\text { No.of } \\
\text { testing } \\
\text { samples }\end{array}$ & $\begin{array}{c}\text { No. of } \\
\text { classes }\end{array}$ & $\begin{array}{c}\text { Diagnosis } \\
\text { accuracy }\end{array}$ \\
\hline 1 & LCD-SVD & $\begin{array}{c}\text { CRO- } \\
\text { SVM }\end{array}$ & 240 & 80 & 4 & 100 \\
\hline 2 & TDF-FDF & PNN & 240 & 80 & 4 & 94.38 \\
\hline 3 & TDF & $\begin{array}{c}\text { Random } \\
\text { forest }\end{array}$ & 200 & 200 & 4 & 98.04 \\
\hline 4 & HSM & Trippy & 225 & 50 & 8 & 98.675 \\
\hline
\end{tabular}

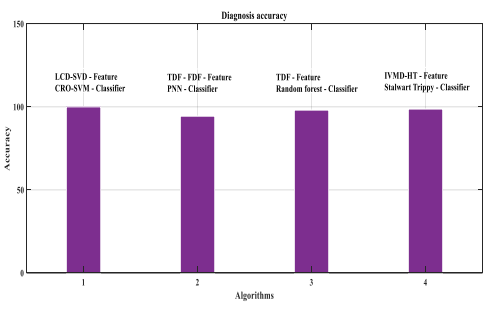

Figure 21: Diagnosis Accuracy of different and our proposed Method

In table 5, that depicts the preparation and testing tests for accurate feature selection utilizing distinctive classes for features extraction. By and large point of view our proposed highlight determination procedure achieve the higher accuracy for choosing the component on the grounds that the preparation informational index are immense contrasted with existing works, for example, LCD-SVD, TDF-FDF and TDF which is effectively feasible to separate the element. In any case, our proposed highlight such RMS, Crest factor and Kurtosis, which are not effectively achievable to remove the component but rather in this work our proposed calculation to extricate the precise required element effortlessly with the assistance of stochastic nature. At long last our proposed diagnosing accuracy esteem is being expanded 98.28 when contrasted with all other existing classifier like CRO, PNN, and Random forest.

\subsubsection{Comparison of diagnosis noise}

Diagnosis Noise is most easily defined via the mean squared error (MSE). Given a noise-free $m \times n$ monochrome feature I and its noisy approximation K, MSE is defined as:

$$
M S E=\frac{1}{m n} \sum_{i=0}^{m-1} \sum_{j=0}^{n-1}[I(i, j)-k(i, j)]^{2}
$$

PSNR (in $\mathrm{dB}$ ) is defined as, 


$$
P S N R=20 \cdot \log _{10}\left(M A X_{I}\right)-10 \cdot \log _{10}(M S E)
$$

Here, $\mathrm{MAX}_{\mathrm{I}}$ is the greatest conceivable blunder estimation of the vibrated signal. At the point when the issues are spoken to utilizing 8 bits for each example, this is 255 . All the more for the most part, when tests are spoken to utilizing direct PCM with B bits per test.

TABLE 5 COMPARISON OF DIAGNOSIS RESULTS UNDER DIFFERENT NOISE ENVIRONMENT

\begin{tabular}{|l|l|l|l|l|l|l|l|l|l|l|}
\hline $\begin{array}{l}\text { Diagonsis } \\
\text { Classifier }\end{array}$ & $12 \mathrm{~dB}$ & $\begin{array}{l}13 \\
\mathrm{~dB}\end{array}$ & $\begin{array}{l}14 \\
\mathrm{~dB}\end{array}$ & $\begin{array}{l}15 \\
\mathrm{~dB}\end{array}$ & $\begin{array}{l}16 \\
\mathrm{~dB}\end{array}$ & $\begin{array}{l}18 \\
\mathrm{~dB}\end{array}$ & $\begin{array}{l}20 \\
\mathrm{~dB}\end{array}$ & $\begin{array}{l}22 \\
\mathrm{~dB}\end{array}$ & $\begin{array}{l}24 \\
\mathrm{~dB}\end{array}$ & $\begin{array}{l}26 \\
\mathrm{~dB}\end{array}$ \\
\hline $\begin{array}{l}\text { Random } \\
\text { Forest }\end{array}$ & 74.78 & 85.82 & 92.95 & 95.34 & 97.74 & 98.60 & 99.00 & 99.26 & 99.46 & 99.53 \\
\hline ELM & 65.53 & 69.53 & 73.01 & 76.39 & 79.72 & 85.48 & 91.40 & 96.81 & 98.83 & 99.37 \\
\hline PNN & 63.25 & 71.64 & 79.16 & 84.84 & 88.77 & 93.43 & 96.51 & 97.81 & 98.28 & 98.87 \\
\hline SVM & 66.87 & 73.32 & 78.85 & 83.59 & 87.66 & 92.28 & 95.68 & 97.24 & 98.11 & 98.67 \\
\hline Proposed & 74.12 & 77.12 & 81.56 & 85.78 & 89.23 & 95.65 & 98.45 & 98.95 & 99.45 & 99.84 \\
\hline
\end{tabular}

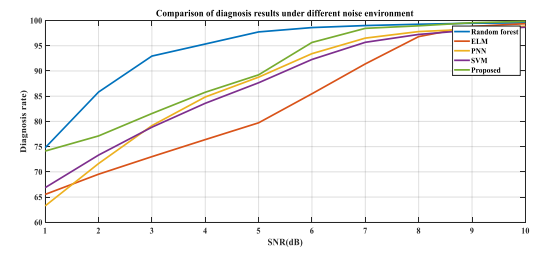

Figure 22: Diagnostic results of employed classifiers with different SNRs.

In the above fig 21 that is clear to seen that Stalwart Trippy Classifier accomplishes factually critical prevail upon the relative techniques when dealing with the test tests with various levels of commotion. As indicated esteems in Table 4, for ELM, PNN ,SVM and stalwart Trippy, a conspicuous descending pattern along these lines shows up when the SNR is lower than $22 \mathrm{~dB}$, while stalwart Trippy still performs fundamentally well in a more extensive scope of SNR attributable to the solid enemy of clamor capacity of our proposed calculation.

\subsubsection{Time consumption of prediction in different classifiers}

Time consumption of prediction is the proportion of aggregate time taken for expectation to the time taken for finishing.

$$
\text { TimeConsumption }=\frac{\text { Time taken to predict the features }}{\text { Total time taken for the completion of the process }}
$$

TABLE 6

TIME CONSUMPTION FOR PREDICTION IN DIFFERENT CLASSIFIERS

\begin{tabular}{|l|l|l|}
\hline Sl.no & Algorithm & $\begin{array}{l}\text { Prediction } \\
\text { Time }\end{array}$ \\
\hline 1 & Random forest & $5.08 \mathrm{~ms}$ \\
\hline 2 & ELM & $0.60 \mathrm{~ms}$ \\
\hline 3 & PNN & $8.44 \mathrm{~ms}$ \\
\hline 4 & SVM & $0.50 \mathrm{~ms}$ \\
\hline 5 & Proposed & $0.45 \mathrm{~ms}$ \\
\hline
\end{tabular}

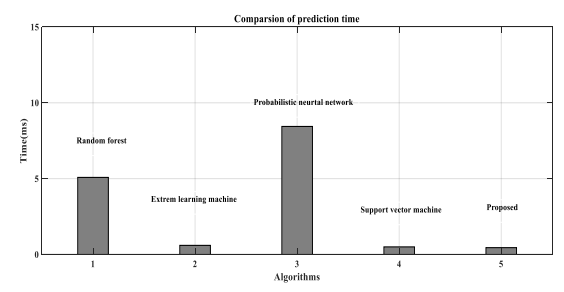

Figure 23:Time consumption of prediction in different classifiers

In the above fig:23 that portrays the general diagnosing forecast time contrasted and existing work and our proposed Stalwart Trippy Classifier accomplish the less expectation time utilizing extensive informational collection for preparing and testing. The upside of our proposed classifier to satisfy in slightest time expectation in blame diagnosing in the turning apparatus. In table 6 esteems showed the expectation time examination of various technique, for example, Random Forest accomplishes the forecast esteem 5.08ms,ELM strategy achieve $0.60 \mathrm{~ms}$,PNN forecast time utilization esteem is $8.44 \mathrm{~ms}$, the forecast of blame diagnosing time SVM technique is $0.50 \mathrm{~ms}$ and our proposed technique RHSM with Stalwart Trippy Algorithm accomplishes the less expectation time because of the specific prescient nature of trippy classifier, which achieve $0.45 \mathrm{~ms}$ forecast time when contrasted with existing our proposed methodology possesses accomplish the better expectation energy for blame determination. Thusly our proposed calculation consequently identify the blame in the pivoting vibrated machine in beginning period and furthermore spares industry from substantial misfortunes happening because of machine breakdowns.

\section{CONCLUSION}

This work builds up a methodology for fault diagnosis for pivoting hardware by using RHSM basedStalwartTrippy classifier. RHSM investigations the precise powerful fault features though the stalwart trippy classifier predicts blame event specifically by methods for extracted faulty features. 
Formally, the above techniques are effectively executed on an air blower and can distinguish the assigned shortcomings with fantastic exactness. Indeed, the blame acknowledgment was finished by examining vibrated signals with computation time of $0.019 \mathrm{sec}, 98.675 \%$ accuracy and prediction time of $0.045 \mathrm{~ms}$ utilizing our organized feature extraction $\mathrm{n}$ and grouping structure.

\section{REFERENCES}

[1] Riaz, Saleem, Hassan Elahi, KashifJavaid, and TufailShahzad, "Vibration feature extraction and analysis for fault diagnosis of rotating machineryA literature survey," Asia Pacific Journal of Multidisciplinary Research, vol. 5, no. 1, pp. 103-110, 2017.

[2] Zhang, XiaoLi, Wei Chen, BaoJian Wang, and XueFeng Chen, "Intelligent fault diagnosis of rotating machinery using support vector machine with ant colony algorithm for synchronous feature selection and parameter optimization," Neurocomputing, vol. 167, pp. 260-279, 2015.

[3] El-Thalji, Idriss, and ErkkiJantunen, "A summary of fault modelling and predictive health monitoring of rolling element bearings," Mechanical Systems and Signal Processing, vol. 60, pp. 252-272, 2015.

[4] V.N. Patel, N. Tandon, and R. K. Pandey, "Vibrations generated by rolling element bearings having multiple local defects on races," Procedia Technology, vol. 14, pp. 312-319, 2014.

[5] P.E. William, M.W. Hoffman, "Identification of bearing faults using time domain zero-crossings. Mech. Syst," Signal Process., vol. 25, pp. 30783088, 2011.

[6] X. Zhang, Y. Liang, J. Zhou, Y. Zang, “A novel bearing fault diagnosis model integrated permutation entropy," ensemble empirical mode decomposition and optimized svm. Measurement, vol. 69, pp. 164-179, 2015.

[7] L. Jiang, H. Yin, X. Li, S. Tang, "Fault diagnosis of rotating machinery based on multi-sensor information fusion using SVM and time-domain features," Shock Vib., pp. 1-8, 2014.

[8] R. Yuan, Y. Lv, G. Song, "Multi-fault diagnosis of rolling bearings via adaptive projection intrinsically transformed multivariate empirical mode decomposition and high order singular value decomposition," Sensors, vol. 18, pp. 1210, 2018.
[9] R. Tiwari, V.K. Gupta, P.K. Kankar, "Bearing fault diagnosis based on multi-scale permutation entropy and adaptive neuro fuzzy classifier," $J$. Vib. Control, vol. 21, pp. 461-467, 2013.

[10] H. Gao, L. Liang, X. Chen, G. Xu, "Feature extraction and recognition for rolling element bearing fault utilizing short-time fourier transform and non-negative matrix factorization," Chin. J. Mech. Eng., vol. 28, pp. 96$105,2015$.

[11] H. Cao, F. Fan, K. Zhou, Z. He, "Wheel-bearing fault diagnosis of trains using empirical wavelet transform," Measurement, vol. 82, pp. 439-449, 2016.

[12] H. Yin, S. Yang, X. Zhu, S. Jin, X. Wang, "Satellite fault diagnosis using support vector machines based on a hybrid voting mechanism," Sci. World J., 2014.

[13] Vapnik, N. Vladimir, "The nature of statistical learning theory," IEEE Trans. Neural Netw., vol. 38, pp. 409, 1997.

[14] S. Bououden, M. Chadli, H.R. Karimi, "An ant colony optimizationbased fuzzy predictive control approach for nonlinear processes," Inf. Sci., vol. 299, pp.143-158, 2015.

[15] S. Bououden, M. Chadli, F. Allouani, S. Filali. "A new approach for fuzzy predictive adaptive controller design using particle swarm optimization algorithm," Int. J. Innovative Comput. Inf. Control., vol. 9, pp. 3741-3758, 2013.

[16] L. Li, M. Chadli, S.X Ding, J. Qiu, Y. Yang, "Diagnostic observer design for $\mathrm{t}-\mathrm{s}$ fuzzy systems: Application to real-time-weighted fault-detection approach," IEEE Trans. Fuzzy Syst., vol. 26, pp. 805-816, 2018.

[17] T. Youssef, M. Chadli, H. R Karimi, R. Wang, "Actuator and sensor faults estimation based on proportional integral observer for ts fuzzy model," J. Franklin Inst., vol. 354, pp. 2524-2542, 2017.

[18] Xie, Yuan, and Tao Zhang, "Fault Diagnosis for Rotating Machinery Based on Convolutional Neural Network and Empirical Mode Decomposition," Shock and Vibration, 2017.

[19] Lv, Yong, Rui Yuan, Tao Wang, Hewenxuan Li, and Gangbing Song, "Health degradation monitoring and early fault diagnosis of a rolling bearing based on CEEMDAN and improved MMSE," Materials, vol. 11, no. 6, pp. 1009, 2018.

[20] Liang, Mingliang, Dongmin Su, Daidi Hu, and MingtaoGe, "A Novel Faults Diagnosis Method for Rolling Element Bearings Based on ELCD and Extreme Learning Machine," Shock and Vibration, 2018.

[21] Pang, Bin, Guiji Tang, TianTian, and Chong Zhou, "Rolling Bearing Fault Diagnosis Based on an Improved HTT Transform," Sensors, vol. 18, no. 4, pp. 1203, 2018.

[22] Li, Chuanhao, Wei Zhang, GaoliangPeng, and Shaohui Liu, "Bearing fault diagnosis using fully-connected winner-take-all autoencoder," IEEE Access, vol. 6, pp. 6103-6115, 2018.

\section{Creative Commons Attribution License 4.0} (Attribution 4.0 International, CC BY 4.0)

This article is published under the terms of the Creative Commons Attribution License 4.0 https://creativecommons.org/licenses/by/4.0/deed.en US 